\title{
Clinical Impact of Pre-transplant Antibodies Against Angiotensin II Type I Receptor and Major Histocompatibility Complex Class I-Related Chain A in Kidney Transplant Patients
}

\begin{abstract}
Ji Won Min, M.D. ${ }^{1 *}$, Hyeyoung Lee, M.D. ${ }^{2,3 *}$, Bum Soon Choi, M.D. ${ }^{4}$, Cheol Whee Park, M.D. ${ }^{4}$, Chul Woo Yang, M.D. ${ }^{4}$,

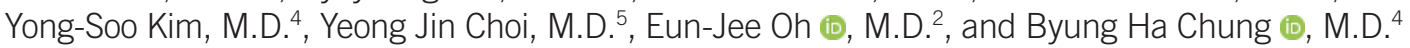

Division of Nephrology, Department of Internal Medicine' ${ }^{1}$, Bucheon St. Mary's Hospital, College of Medicine, The Catholic University of Korea, Bucheon; Department of Laboratory Medicine ${ }^{2}$, Seoul St. Mary's Hospital, College of Medicine, The Catholic University of Korea, Seoul; Department of Laboratory Medicine $^{3}$, Catholic Kwandong University International St. Mary's Hospital, Incheon; Division of Nephrology, Department of Internal Medicine ${ }^{4}$, Department of Hospital Pathology ${ }^{5}$, Seoul St. Mary's Hospital, College of Medicine, The Catholic University of Korea, Seoul, Korea
\end{abstract}

Background: Evidence of antibody-mediated injury in the absence of donor-specific HLA antibodies (HLA-DSA) has recently emerged, suggesting a role of antibodies in targeting non-HLA antigens expressed on renal allograft tissue. However, the clinical significance of pre-transplant non-HLA antibodies remains unclear. We compared the histological and clinical impact of pre-transplant HLA-DSA and non-HLA antibodies, especially angiotensin II type I receptor (anti-AT1R) and MHC class I-related chain A (anti-MICA), in kidney transplant patients.

Methods: Pre-transplant HLA-DSA, anti-AT1R, and anti-MICA were retrospectively examined in 359 kidney transplant patients to determine the effect of each antibody on allograft survival and clinical characteristics.

Results: Pre-transplant HLA-DSA, anti-AT1R, and anti-MICA were detected in 37 (10.3\%), 174 (48.5\%), and 50 patients (13.9\%), respectively. Post-transplant antibody-mediated rejection was associated with a pre-transplant HLA-DSA (+) status only. The development of microvascular inflammation (MVI) was associated with pre-transplant HLA-DSA $(P=0.001)$ and anti-AT1R $(P=0.036)$. Anti-AT1R $(+)$ patients had significantly lower allograft survival compared with anti-AT1R ( - ) patients $(P=0.042)$. Only pre-transplant anti-AT1R positivity was an independent risk factor for allograft failure (hazard ratio 4.824 , confidence interval 1.017-24.888; $P=0.038$ ). MVI was the most common histological feature of allograft failure in patients with pre-transplant anti-AT1R.

Conclusions: Pre-transplant anti-AT1R is an important risk factor for allograft failure, which may be mediated by MVI induction in the allograft tissue.

Key Words: Angiotensin II type 1 receptor, Donor-specific anti-HLA antibody, Microvascular inflammation, Kidney transplantation
Received: September 25, 2017

Revision received: November 27, 2017

Accepted: May 6, 2018

\section{Corresponding author: Eun-Jee Oh}

(iD https://orcid.org/0000-0001-5870-915X

Department of Laboratory Medicine, Seoul

St. Mary's Hospital, College of Medicine,

The Catholic University of Korea, 222

Banpo-daero, Seocho-gu, Seoul 06591,

Korea

Tel: +82-2-2258-1641

Fax: +82-2-2258-1719

E-mail: ejoh@catholic.ac.kr

Co-corresponding author: Byung Ha Chung (ib) https://orcid.org/0000-0003-0048-5717

Department of Internal Medicine, Seoul St. Mary's Hospital, College of Medicine,

The Catholic University of Korea, 222 Banpo-daero, Seocho-gu, Seoul 06591,

Korea

Tel: +82-2-2258-6066

Fax: +82-2-536-0323

E-mail: chungbh@catholic.ac.kr

*These authors contributed equally to this work.

(C) Korean Society for Laboratory Medicine This is an Open Access article distributed under the terms of the Creative Commons Attribution Non-Commercial License (http://creativecommons.org/licenses/by-nc/4.0) which permits unrestricted non-commercial use, distribution, and reproduction in any medium, provided the original work is properly cited. 


\section{INTRODUCTION}

There is now ample evidence of an association between preformed antibody to HLA and vascular rejection in kidney transplantation (KT) [1-3]. A recent study demonstrated the possibility of antibody-mediated injury without donor-specific HLA antibody (HLADSA) production, suggesting a role of antibodies against nonHLA antigens expressed on the renal allograft tissue [4].

Among the various types of non-HLA antibodies, angiotensin II type 1 receptor antibodies (anti-AT1Rs) and the MHC class Irelated chain $A$ antibodies (anti-MICAs) have been the most widely studied $[5,6]$. AT1R is a G-protein-coupled receptor that mediates the actions of angiotensin II, including blood pressure regulation and water-salt balance, and anti-AT1Rs may be formed as a result of inflammation, injury, sensitization, or non-compliance to medication [7]. Polymorphic MICA molecules are constitutively expressed on vascular endothelial cells, and previous experiments revealed that increased MICA expression levels were associated with hypoxic stress conditions accompanying transplantation [8]. Although the adverse effects of anti-AT1Rs or anti-MICA on allograft outcomes have been demonstrated in several studies [9-14], the clinical significance of pre-transplant non-HLA antibodies remains controversial, and studies on the association between non-HLA antibodies and histological outcomes are limited.

We investigated the comparative impact of the presence of pre-transplant non-HLA antibodies (anti-AT1Rs and anti-MICAs) and HLA-DSA on clinical and histological outcomes in KT patients.

\section{METHODS}

\section{Study population}

Between March 2010 and September 2014, a total of 475 patients received KT at Seoul St. Mary's Hospital, Korea. Among them, 116 patients were excluded for the following reasons: 65 ABO-incompatible KT patients, five patients who died with a functioning graft, three patients who underwent simultaneous $\mathrm{KT}$ and bone marrow transplantation, one patient who underwent simultaneous $\mathrm{KT}$ and pancreas transplantation, and 42 patients without available pre-transplant serum samples. Therefore, a total of 359 patients were finally enrolled in this study, and 253 were subjected to either protocol $(N=121)$ or indication biopsy ( $\mathrm{N}=132)$. The median follow-up duration for graft survival was 32.0 (95\% confidence interval [Cl]: 29.5-35.0) months. Patients' baseline characteristics are presented in Table 1.
Table 1. Baseline characteristics of 359 kidney transplant patients

\begin{tabular}{|c|c|}
\hline Age at transplantation (yr), mean \pm SD & $47.3 \pm 11.7$ \\
\hline Gender; male, N (\%) & $198(55.2)$ \\
\hline HLA mismatch number & $2.94 \pm 1.83$ \\
\hline Re-transplantation & $38(10.6)$ \\
\hline Deceased donor, N (\%) & $87(24.2)$ \\
\hline \multicolumn{2}{|l|}{ Desensitization, N (\%) } \\
\hline Rituximab & $50(13.9)$ \\
\hline Rituximab+Plasmapheresis & $31(8.6)$ \\
\hline \multicolumn{2}{|l|}{ Induction therapy, N (\%) } \\
\hline Basiliximab & $299(83.3)$ \\
\hline ATG & $60(16.7)$ \\
\hline \multicolumn{2}{|l|}{ Maintenance therapy, N (\%) } \\
\hline Tac+MMF+steroid & $336(93.7)$ \\
\hline CsA+MMF+steroid & $23(6.3)$ \\
\hline \multicolumn{2}{|l|}{ High PRA (> 50\%), N (\%) } \\
\hline Total & $92(25.6)$ \\
\hline Class I & $59(16.4)$ \\
\hline Class II & $58(16.2)$ \\
\hline \multicolumn{2}{|l|}{ HLA-DSA (+), N (\%) } \\
\hline Total & $37(10.3)$ \\
\hline Class I & $30(8.4)$ \\
\hline Class II & $15(4.2)$ \\
\hline Non-HLA antibodies (+), N (\%) & $202(56.3)$ \\
\hline Anti-AT1R (+) & $174(48.5)$ \\
\hline - Anti-AT1R only (+) & $152(42.3)$ \\
\hline Anti-MICA (+) & $50(13.9)$ \\
\hline - Anti-MICA only (+) & $28(7.8)$ \\
\hline Both anti-AT1R and anti-MICA (+) & $22(6.1)$ \\
\hline
\end{tabular}

Abbreviations: ATG, anti-thymocyte globulin; Tac, tacrolimus; MMF, mycophenolate mofetil; CsA, cyclosporin A; PRA, panel reactive antibody; DSA, donor-specific antibody; anti-AT1R, angiotensin II type 1 receptor antibody; anti-MICA, MHC class I-related chain A antibody.

This study was approved by the Institutional Review Board of Seoul St. Mary's Hospital (KC13TNMI0701). All patients provided written informed consent.

\section{Pre-transplant immunologic work-up and immunosuppressive regimen}

Pre-transplant immunologic workup was performed as described previously [15]. In brief, we performed panel reactive antibody (PRA)-Luminex screening and cross-match (XM) testing using complement-dependent cytotoxicity (CDC), CDC-anti-human globulin, and flow cytometric XM in all patients. In patients with 
positive PRA or positive XM test results, we investigated the presence of HLA-DSA using a Luminex single-antigen assay (One Lambda, Canoga Park, CA, USA). Desensitization therapy was performed on living donor transplant patients who tested positive for pre-transplant HLA-DSA and/or T cell or B cell XM, as described previously [15]. In brief, rituximab (RTX) at a dose of 375 mg/m² (MabThera Genentech, Inc., San Francisco, CA, USA) was administered two to three weeks before transplantation, and plasmapheresis/intravenous immunoglobulin (PP/IVIG) therapy was initiated 13 days prior to transplantation and administered every 48 hours. Tacrolimus (Tac) or cyclosporin A (CsA) was administered in combination with mycophenolate mofetil and prednisolone as a maintenance immunosuppressant (IS) regimen, with Tac as the main IS in all HLA-DSA (+) patients regardless of desensitization and median fluorescent intensity (MFI) strength. Basiliximab or anti-thymocyte globulin was administered as induction therapy.

\section{Detection of anti-AT1Rs and anti-MICAs}

Pre-transplant anti-AT1R and anti-MICA was assessed for all 359 patients, using serum samples taken before the initiation of IS. Serum anti-AT1R levels were measured using the EIA-AT1R kit (One Lambda). Microtiter 96-well polystyrene plates were coated with AT1R, and $100 \mu \mathrm{L}$ of diluted samples were incubated at $2-8^{\circ} \mathrm{C}$ for 2 hours according to the manufacturer instructions. After washing, the plates were incubated for $60 \mathrm{~min}$ utes with $100 \mu \mathrm{L}$ horseradish peroxidase-labeled anti-human IgG. After incubation with $100 \mu \mathrm{L}$ tetramethylbenzidine substrate for 20 minutes, the optical absorbance of each well was measured at $450 \mathrm{~nm}$. Concentrations of anti-AT1Rs were determined based on the calibration curve, in which $10 \mathrm{U} / \mathrm{mL}$ was considered to be the cut-off value following the manufacturer's recommendation, similar to some previous studies $[9,16]$. AntiMICA was measured using the LABScreen Mixed assay (One Lambda) on a Luminex platform according to the manufacturer's specifications. MICA alleles *001, *002, *004, *007, *009, ${ }^{*} 012,{ }^{*} 017,{ }^{*} 018,{ }^{*} 019$, and ${ }^{*} 027$ were coated in microbeads.

\section{Histological examination of renal allograft biopsy}

Protocol biopsy was performed at three months post-transplant in 121 patients after obtaining consent. Indication biopsy was performed in patients with a $20 \%$ increase in serum creatinine above the baseline value at any time post-transplant. The mean time to indication biopsy was $5.7 \pm 9.8$ months. The biopsies were performed as described in our previous study [17], using a 16-gauge biopsy gun under ultrasonic localization. Histopath- ological diagnosis was made based on the revised Banff working classification [18]. The microvascular inflammation (MVI) score was defined as the sum of the glomerulitis (g) and peritubular capillaritis (ptc) scores. The threshold for moderate MVI (g + ptc $\geq 2$ ) was used to determine the association of antibodies with $\mathrm{MVI}$, which has been applied for the diagnosis of $\mathrm{C} 4 \mathrm{~d}(-)$ acute antibody-mediated rejection (ABMR) in the revised Banff criteria [18].

\section{Statistical analysis}

Continuous variables are presented as mean $\pm \mathrm{SD}$, and categorical variables are summarized as number and percentages. Continuous variables were compared using the Student's t-test, and categorical variables were compared using the chi-square test or Fisher's exact test. Allograft survival among groups was compared using Kaplan-Meier analysis with a log-rank test. Risk factors for MVI were determined by odds ratios (OR) using a multivariable logistic regression analysis. Risk factors affecting allograft outcomes were determined by hazard ratios (HR) using a Cox regression multivariate analysis. Statistical analysis was performed using SPSS version 20 for Windows (SPSS Inc., Chicago, IL, USA) and MedCalc version 15.5 (MedCalc, Mariakerke, Belgium). $P<$ 0.05 was considered statistically significant.

\section{RESULTS}

\section{Prevalence of HLA-DSA, anti-AT1R, and anti-MICA in pre- transplant sera}

As shown in Table 1, pre-transplant HLA-DSA was detected in 37 of the $359 \mathrm{KT}$ patients (10.3\%), with more patients producing class I than class II. Moreover, pre-transplant anti-AT1R was detected in 174 patients (48.5\%), and pre-transplant anti-MICA was detected in only 50 patients (13.9\%). Of the 37 HLA-DSA (+) patients, 21 patients (56.8\%) were anti-AT1R (+) and five patients (13.5\%) were anti-MICA (+). Of the 322 HLA-DSA (-) patients, 153 patients $(47.5 \%)$ were anti-AT1R $(+)$ and 45 patients (14\%) were anti-MICA (+).

\section{Association of pre-transplant antibodies with histological diagnosis of allograft biopsy}

There was a higher prevalence of ABMR in the HLA-DSA (+) patients $(9 / 26,34.6 \%)$ compared with that in the HLA-DSA (-) patients $(8 / 227,3.5 \%)(P<0.001)$. However, no significant difference in ABMR frequencies was detected between pre-transplant anti-AT1R (+) and (-) patients (10/126 [7.9\%] vs 7/127 [5.5\%]) or anti-MICA (+) and (-) patients (3/35 [8.6\%] vs 14/218 
[6.4\%]). In addition, T cell-mediated rejection (TCMR) development was not associated with the presence of pre-transplant HLA-DSA, anti-AT1R, or anti-MICA. The prevalence of MVI was significantly higher not only in HLA-DSA (+) patients compared with HLA-DSA (-) patients (14/26 [53.8\%] vs 34/227 [15.0\%]; $P<0.001)$ but also in anti-AT1R (+) patients compared with antiAT1R (-) patients (31/126 [24.6\%] vs 17/127 [13.4\%]; $P=0.023)$. In HLA-DSA (+) anti-AT1R (-) patients and HLA-DSA (-) antiATIR (+) patients, the mean durations to MVI development were similar at $11.2 \pm 20.1$ months and $8.4 \pm 10.7$ months, respectively. Patients with both HLA-DSA and anti-AT1R showed a relatively earlier onset of MVI (2.1 \pm 3.4 months post-transplant),

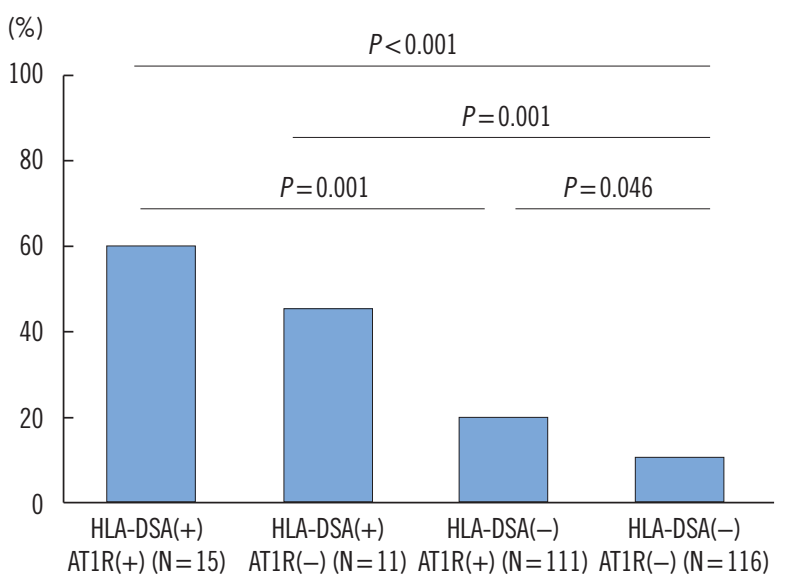

Fig. 1. Synergistic impacts of HLA-DSA and anti-AT1R on MVI. Detection of MVI was the highest in patients with both HLA-DSA and anti-AT1R, showing a synergistic impact $(P<0.001$, chi-square for trend). In patients without HLA-DSA, the presence of anti-AT1R was significantly associated with the development of MVI (12/116 vs 22/111, $P=0.046)$. HLA-DSA increased the incidence of MVI in both patients with $(P=0.001)$ and without $(P=0.001)$ anti-AT1Rs.

Abbreviations: DSA, donor-specific antibody; anti-AT1R, angiotensin II type 1 receptor antibody. but this difference was not statistically significant. However, MVI was detected most frequently in patients with both HLA-DSA and anti-AT1R (Fig. 1; $P<0.001$, chi-square for trend). Among the 48 patients with MVI, 17 (35.4\%) were diagnosed as having ABMR based on the revised Banff criteria. There was also no significant difference in the frequency of MVI development between anti-MICA (+) and anti-MICA (-) patients (9/35 [25.7\%] vs 39/218 [17.9\%]). There were no statistically significant associations of each antibody with individual Banff classification scores (g, ptc, and C4d); however, the anti-AT1R (+) and antiMICA (+) groups also showed a tendency toward higher $g$ and ptc scores, but not C4d scores.

The frequencies of de novo DSA (dn DSA) development were not significantly different between pre-transplant anti-AT1R (+) (7.9\%, 5/63) and anti-AT1R (-) patients (5.2\%, 3/58). In addition, the time to detection of $d n$ DSA did not vary according to the pre-transplant anti-AT1R status. All five patients with dnDSA that were pre-transplant anti-AT1R (+) showed MVI, and three patients showed ABMR.

\section{Multivariate analysis of pre-transplant risk factors for MVI in allograft biopsy}

Among the 253 biopsies analyzed, both anti-AT1R and HLADSA proved to be significant risk factors for MVI (Table 2). When we performed subgroup analysis for the indication and protocol biopsy groups, both HLA-DSA (OR 4.802, 95\% Cl [1.46-15.726]; $P=0.010$ ) and anti-AT1R (OR 2.694, 95\% Cl [1.086-6.678]; $P=0.032$ ) emerged as significant risk factors for MVI in the indication biopsy group. However, in the protocol biopsy subgroup, only HLA-DSA was a significant risk factor for MVI (OR 8.810, 95\% Cl [1.656-46.877]; $P=0.011$ ).

Table 2. Pre-transplant risk factors for MVI in allograft biopsy

\begin{tabular}{|c|c|c|c|c|c|c|}
\hline & & Univariate & & & Multivariate & \\
\hline & $\mathrm{OR}$ & $(95 \% \mathrm{Cl})$ & $P$ & $\mathrm{OR}$ & $(95 \% \mathrm{Cl})$ & $P$ \\
\hline Anti-AT1R & 1.828 & (1.009-3.311) & 0.047 & 2.033 & $(1.049-3.940)$ & 0.036 \\
\hline Anti-MICA & 1.352 & $(0.652-2.802)$ & 0.418 & & & \\
\hline HLA-DSA & 6.958 & (3.371-14.361) & 0.000 & 4.987 & $(1.978-12.572)$ & 0.001 \\
\hline HLA mm & 2.307 & $(0.652-8.161)$ & 0.195 & & & \\
\hline DDKT & 0.934 & $(0.482-1.811)$ & 0.839 & & & \\
\hline Re-transplant & 4.003 & $(0.855-18.737)$ & 0.078 & & & \\
\hline
\end{tabular}

Abbreviations: MVI, microvascular inflammation (MVI score $\geq 2$ ); OR, odds ratio; Cl, confidence interval; anti-AT1R, angiotensin II type 1 receptor antibody; anti-MICA, MHC class I-related chain A antibody; DSA, donor-specific antibody; mm, mismatch; DDKT, deceased donor kidney transplant. 
4. Effects of pre-transplant antibodies on allograft survival rate Patients that were HLA-DSA (+) showed significantly poorer allograft survival compared with HLA-DSA (-) patients (Fig. 2A, $P=0.037)$. Similarly, anti-AT1R (+) patients showed significantly inferior graft survival compared with anti-AT1R (-) patients (Fig. $2 \mathrm{~B}, P=0.042$ ). However, there was no significant difference in graft survival between the anti-MICA (+) and anti-MICA (-) groups (Fig. 2C). There was also no statistically significant synergic effect of HLA-DSA and anti-AT1R on graft survival. Overall survival was poorest in the HLA-DSA(+)AT1R(+) group (Fig. 2D). Nine of the 11 patients who suffered from allograft failure (81\%) were anti-AT1R (+), whereas only two (18\%) were HLA-DSA (+) pretransplant (Table 3). All nine anti-AT1R (+) patients with allograft failure presented with MVI on allograft biopsy, including three patients (no. 7, 8, 9) diagnosed as having ABMR $(33.3 \%)$, and the other six patients exhibited C4d(-) MVI (66.6\%). The average duration to failure of the C4d(-) MVI patients was $24 \pm 11.9$ months. Cox regression multivariate analysis showed that anti-
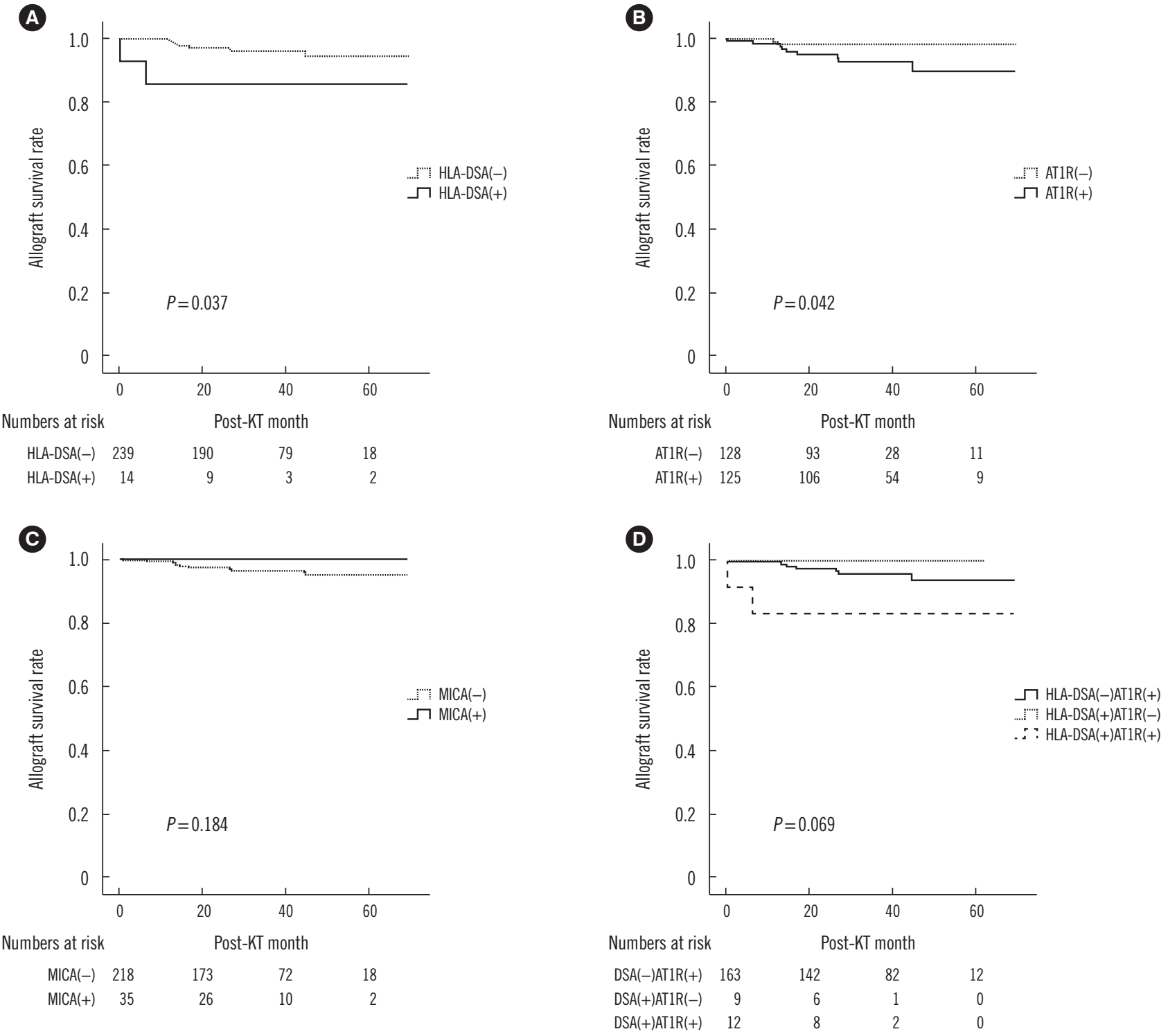

Fig. 2. Comparison of allograft survival. (A) Patients that were HLA-DSA (+) showed significantly lower allograft survival rates $(P=0.037)$, and $(B)$ patients that were anti-AT1R $(+)$ showed significantly lower allograft survival rates $(P=0.042)$. (C) There was no effect of the presence of anti-MICA on allograft survival. (D) The HLA-DSA(-)AT1R(+) group showed a tendency for poorer survival compared with the HLADSA(+)AT1R(-) group. Survival was poorest in the HLA-DSA(+)AT1R(+) group $(P=0.069)$.

Abbreviations: DSA, donor-specific antibody; anti-AT1R, angiotensin II type 1 receptor antibody; anti-MICA, MHC class I-related chain A antibody. 
Table 3. Characteristics of patients with graft failure

\begin{tabular}{|c|c|c|c|c|c|c|c|c|c|c|c|c|c|}
\hline \multirow[b]{2}{*}{ No. Case } & \multicolumn{6}{|c|}{ Pre-transplant } & \multicolumn{7}{|c|}{ Time of Biopsy } \\
\hline & $\begin{array}{l}\text { Sex /Age } \\
\text { (year) }\end{array}$ & $\begin{array}{l}\text { HLA } \\
\text { MM }\end{array}$ & $\begin{array}{l}\text { Anti- } \\
\text { AT1R }\end{array}$ & $\begin{array}{l}\text { Anti- } \\
\text { MICA }\end{array}$ & $\begin{array}{l}\text { HLA- } \\
\text { DSA }\end{array}$ & $\begin{array}{c}\text { Months to } \\
\text { Biopsy }\end{array}$ & $\begin{array}{l}\text { HLA- } \\
\text { DSA }\end{array}$ & Histological Dx & $\begin{array}{l}\text { C4d } \\
\text { score }\end{array}$ & $\begin{array}{l}\text { MVI } \\
\text { score }\end{array}$ & $\begin{array}{c}\mathrm{Cr} \\
(\mathrm{mg} / \mathrm{dL})\end{array}$ & $\begin{array}{c}\text { Treatment for } \\
\text { rejection after biopsy }\end{array}$ & $\begin{array}{l}\text { Months to } \\
\text { graft loss }\end{array}$ \\
\hline 1 & $\mathrm{M} / 69$ & 5 & Y & $\mathrm{N}$ & $\mathrm{N}$ & 11 & $\mathrm{~N}$ & C4d (-) MVI, TCMR & 0 & 4 & 3.12 & MPS, ATG & 13 \\
\hline 2 & M/67 & 3 & Y & $\mathrm{N}$ & $\mathrm{N}$ & 9 & $\mathrm{~N}$ & C4d (-) MVI, TCMR & 0 & 3 & 2.53 & MPS & 27 \\
\hline 3 & $F / 56$ & 3 & Y & $\mathrm{N}$ & N & 5 & $\mathrm{~N}$ & C4d (-) MVI, TCMR & 0 & 2 & 1.92 & MPS & 15 \\
\hline 4 & $F / 52$ & 3 & Y & N & $\mathrm{N}$ & 40 & $\mathrm{~N}$ & C4d (-) MVI & 0 & 4 & 2.93 & None & 45 \\
\hline 5 & $F / 73$ & 5 & Y & N & $\mathrm{N}$ & 2 & $\mathrm{~N}$ & C4d (-) MVI & 0 & 2 & 1.47 & None & 17 \\
\hline 6 & $\mathrm{M} / 60$ & 4 & Y & N & $\mathrm{N}$ & 3 & $\mathrm{~N}$ & C4d (-) MVI & 0 & 3 & 1.71 & None & 27 \\
\hline 7 & M/64 & 5 & Y & N & $\mathrm{N}$ & 10 & Y & C4d (-) ABMR & 0 & 5 & 4.05 & MPS, PP/IVIG & 14 \\
\hline 8 & $M / 41$ & 5 & Y & $\mathrm{N}$ & Y & 0.2 & Y & ABMR & 3 & 2 & 1.41 & MPS, PP/IVIG, RTX & 7 \\
\hline 9 & $F / 34$ & 5 & Y & $\mathrm{N}$ & Y & 0.2 & Y & ABMR & 3 & 4 & 6.98 & MPS, PP/IVIG, RTX & 0.4 \\
\hline 10 & $M / 48$ & 4 & $\mathrm{~N}$ & $\mathrm{~N}$ & $\mathrm{~N}$ & 2 & $\mathrm{~N}$ & TCMR & 0 & 0 & 1.91 & MPS & 13 \\
\hline 11 & $F / 44$ & 3 & $\mathrm{~N}$ & $\mathrm{~N}$ & $\mathrm{~N}$ & 9 & $\mathrm{~N}$ & TCMR & 0 & 0 & 1.34 & MPS, ATG, & 12 \\
\hline
\end{tabular}

Abbreviations: MM, mismatch; DSA, donor-specific antibody; anti-AT1R, angiotensin II type 1 receptor antibody; anti-MICA, MHC class I-related chain A antibody; Cr, serum creatinine; Y, yes; N, no; ABMR, acute antibody-mediated rejection; TCMR, T cell-mediated rejection; MPS, methylprednisolone; ATG, antithymocyte globulin; PP/IVIG, plasmapheresis/intravenous immunoglobulin; RTX, rituximab.

Table 4. Pre-transplant risk factors for allograft failure

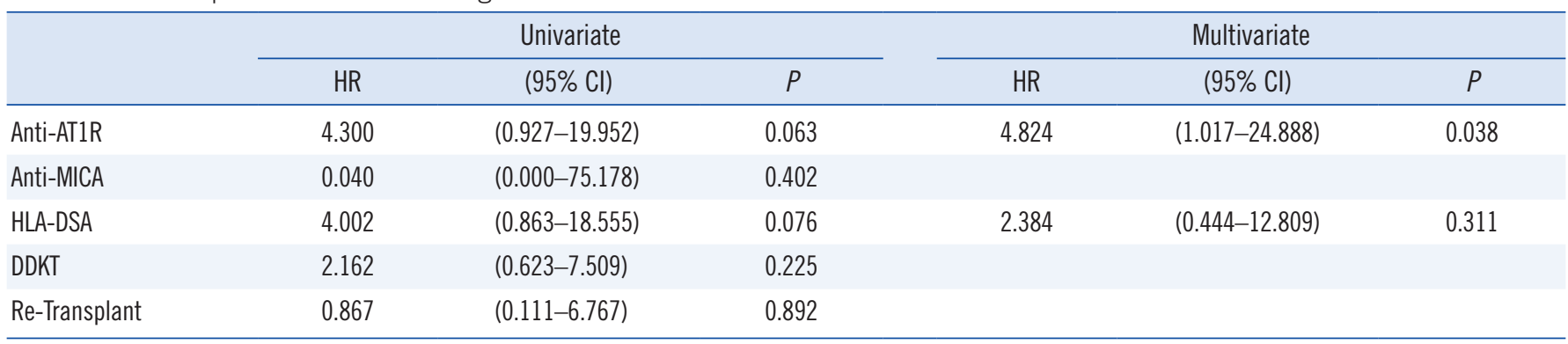

Abbreviations: HR, hazard ratio; Cl, confidence interval; anti-AT1R, angiotensin II type 1 receptor antibody; anti-MICA, MHC class I-related chain A antibody; DSA, donor-specific antibody; DDKT, deceased donor kidney transplant.

AT1R was the only significant risk factor for the development of allograft failure (HR 4.824, 95\% Cl [1.017-24.888], $P=0.038$ ) (Table 4).

\section{DISCUSSION}

Our study demonstrates that pre-transplant anti-AT1R is a significant risk factor for the development of MVI in allograft biopsy, and that it contributes to poor post-KT allograft survival.

First, we investigated the prevalence of each antibody at the pre-transplant phase. Previous reports have shown an incidence of anti-AT1R (+) status in the range of 10.0-47.2\% [7, 9, 11, 19]. We detected pre-transplant anti-AT1R antibodies in $48.5 \%$ of patients, which is similar to the results of Giral et al [9], and to a recent study reporting a $59.0 \%$ rate of anti-AT1R (+) [20].
The incidence of pretransplant anti-MICAs has been reported to be approximately $7.2-25 \%[13,21]$. We could not find any association between the detection of anti-HLA antibodies (HLADSA) and non-HLA antibodies.

Next, we investigated the impact of each antibody at the pretransplant stage on the risk of the post-transplant development of ABMR and MVI. The presence of pre-transplant HLA-DSA is a well-known major risk factor for antibody-mediated allograft injury [5, 15, 22, 23], which was confirmed in the present study. Pre-transplant AT1R detection was an independent risk factor for the development of MVI. Activation by anti-AT1R increases pro-inflammatory protein expression, thereby stimulating the recruitment of inflammatory cells [14, 24, 25]. These processes can result in the development of peritubular capillaritis and glomerulitis. Moreover, endothelial cell damage caused by anti-AT1R 
is not mediated by complement activation but rather through ERK1/2 followed by AP-1 and NF-kB, resulting in the activation of MCP-1 and RANTES and in the up-regulated expression of the tissue factor $[6,26,27]$. Therefore, C4d deposition in the peritubular capillary is rarely detected in these cases, and the ABMR due to anti-AT1R presents most often as C4d(-) MVI, which was detected in the present study (Table 3).

Interestingly, we also found that MVI was most frequently detected in patients with both HLA-DSA and anti-AT1R, suggesting the synergistic impact of the two antibodies; it supports previous reports showing that HLA-DSA and non-HLA antibodies may function in synergy $[11,14]$. An explanation for this synergistic impact may be that the pro-inflammatory environment induced either by HLA-DSA or anti-AT1R can encourage further up-regulation of AT1R or HLA expression [14, 24, 25]. In our study, there was no difference in the time to detection of $d n D S A$ at the time of indication biopsy with respect to the pre-transplant anti-AT1R status. This is in contrast to the findings of Cuevas et al [28], who reported that a high pre-transplant anti-AT1R level was an independent risk factor for earlier dnDSA detection after KT. Further follow-up studies are needed to clarify the association between pre-transplant anti-AT1R (+) status and dnDSA development.

Finally, we investigated the impact of each antibody on posttransplant allograft survival rates, and found that recipients with pre-transplant anti-AT1R showed poorer allograft survival. However, HLA-DSA did not emerge as a significant risk factor for allograft failure in our study. The detrimental effects of preformed HLA-DSA are well-known; therefore, desensitization therapies are initiated in patients when pre-transplant HLA-DSA is detected $[15,29]$. Moreover, in patients that are HLA-DSA $(+)$ at the pretransplant stage, MVI usually presents as early ABMR, combined with rapid deterioration of allograft function. Therefore, early intervention would be performed in these patients along with desensitization therapy [30, 31]. In contrast, in patients that are pre-transplant anti-AT1R (+), detection of C4d (-) MVI without HLA-DSA at the time of biopsy could not be diagnosed as ABMR. In addition, allograft injury due to anti-AT1R occurs via a chronic damaging process, and the clinical course is usually indolent [4]. Therefore, aggressive therapy is not performed in such cases, which might result in the progression of chronic allograft injury leading to poorer allograft outcomes.

In contrast to anti-AT1R, anti-MICA did not show a significant impact on any post-transplant outcome, supporting a previous study, which suggested that the role of anti-MICA was questionable under sufficient immune suppression [32]. The current practice guideline does not recommend routine typing for antiMICA in KT patients [33]. Further studies including MICA genotyping and determination of donor specificity are needed to clarify these effects.

The main limitation of this study is its retrospective cohort design. Therefore, we were not able to measure non-HLA antibody levels in the patients at the time of allograft biopsy. Given that a previous study found an association of anti-AT1R at the time of biopsy with antibody-mediated injury [34], further randomized controlled studies on the monitoring of anti-AT1R antibodies are required to reinforce our findings.

In conclusion, anti-AT1R, but not anti-MICA, detected before KT may be an important risk factor for allograft failure, which could be mediated by the induction of MVI in the allograft tissue. Hence, development of $\mathrm{C} 4 \mathrm{~d}(-) \mathrm{MVI}$ might require more aggressive anti-humoral therapy in patients with pre-transplant anti-AT1R.

\section{Authors' Disclosures of Potential Conflicts of Interest}

The authors have no conflicts of interest to disclose.

\section{Acknowledgment}

This research was supported by a grant from the Korean Health Technology R\&D Project, Ministry of Health \& Welfare, Republic of Korea (HI13C1232), and by the research fund of Seoul St. Mary's Hospital, The Catholic University of Korea.

\section{REFERENCES}

1. Terasaki PI and Ozawa M. Predicting kidney graft failure by HLA antibodies: a prospective trial. Am J Transplant 2004;4:438-43.

2. Loupy A, Lefaucheur C, Vernerey D, Prugger C, Duong van Huyen JP, Mooney N, et al. Complement-binding anti-HLA antibodies and kidneyallograft survival. N Engl J Med 2013;369:1215-26.

3. Wiebe C, Gibson IW, Blydt-Hansen TD, Karpinski M, Ho J, Storsley LJ, et al. Evolution and clinical pathologic correlations of de novo donor-specific HLA antibody post kidney transplant. Am J Transplant 2012;12:115767.

4. Opelz G and Collaborative Transplant Study. Non-HLA transplantation immunity revealed by lymphocytotoxic antibodies. Lancet 2005;365: 1570-6.

5. Tait BD, Susal C, Gebel HM, Nickerson PW, Zachary AA, Claas FH, et al. Consensus guidelines on the testing and clinical management issues associated with HLA and non-HLA antibodies in transplantation. Transplantation 2013;95:19-47.

6. Dragun D, Muller DN, Brasen JH, Fritsche L, Nieminen-Kelha M, Dechend $\mathrm{R}$, et al. Angiotensin II type 1-receptor activating antibodies in re- 
nal-allograft rejection. N Engl J Med 2005;352:558-69.

7. Reinsmoen NL, Lai CH, Heidecke H, Haas M, Cao K, Ong G, et al. Antiangiotensin type 1 receptor antibodies associated with antibody mediated rejection in donor HLA antibody negative patients. Transplantation 2010;90:1473-7.

8. Luo L, Lu J, Wei L, Long D, Guo JY, Shan J, et al. The role of HIF-1 in up-regulating MICA expression on human renal proximal tubular epithelial cells during hypoxia/reoxygenation. BMC Cell Biol 2010;11:91.

9. Giral M, Foucher Y, Dufay A, Van Huyen JP, Renaudin K, Moreau A, et al. Pretransplant sensitization against angiotensin II type 1 receptor is a risk factor for acute rejection and graft loss. Am J Transplant 2013;13: 2567-76.

10. Sanchez-Zapardiel E, Castro-Panete MJ, Mancebo E, Morales P, Laguna-Goya R, Morales JM, et al. Early renal graft function deterioration in recipients with preformed anti-MICA antibodies: partial contribution of complement-dependent cytotoxicity. Nephrol Dial Transplant 2016;31: 150-60.

11. Taniguchi M, Rebellato LM, Cai J, Hopfield J, Briley KP, Haisch CE, et al. Higher risk of kidney graft failure in the presence of anti-angiotensin II type-1 receptor antibodies. Am J Transplant 2013;13:2577-89.

12. Lee J, Park Y, Kim BS, Lee JG, Kim HJ, Kim YS, et al. Clinical implications of angiotensin II type 1 receptor antibodies in antibody-mediated rejection without detectable donor-specific HLA antibodies after renal transplantation. Transplant Proc 2015;47:649-52.

13. Zou Y, Stastny P, Susal C, Dohler B, Opelz G. Antibodies against MICA antigens and kidney-transplant rejection. N Engl J Med 2007;357:1293300.

14. Philogene MC, Bagnasco S, Kraus ES, Montgomery RA, Dragun D, Leffell MS, et al. Anti-angiotensin II type 1 receptor and anti-endothelial cell antibodies: a cross-sectional analysis of pathological findings in allograft biopsies. Transplantation 2017; 101:608-15.

15. Chung BH, Choi BS, Oh EJ, Park CW, Kim JI, Moon IS, et al. Clinical impact of the baseline donor-specific anti-human leukocyte antigen antibody measured by Luminex single antigen assay in living donor kidney transplant recipients after desensitization therapy. Transpl Int 2014;27: 49-59.

16. Fuss A, Hope CM, Deayton S, Bennett GD, Holdsworth R, Carroll RP, et al. C4d-negative antibody-mediated rejection with high anti-angiotensin II type I receptor antibodies in absence of donor-specific antibodies. Nephrology (Carlton) 2015;20:467-73.

17. Chung BH, Lee JY, Kang SH, Sun IO, Choi SR, Park HS, et al. Comparison of clinical outcome between high and low baseline anti-ABO antibody titers in ABO-incompatible kidney transplantation. Ren Fail 2011; 33:150-8.

18. Haas M, Sis B, Racusen LC, Solez K, Glotz D, Colvin RB, et al. Banff 2013 meeting report: inclusion of c4d-negative antibody-mediated rejection and antibody-associated arterial lesions. Am J Transplant 2014; 14:272-83.

19. Banasik M, Boratynska M, Koscielska-Kasprzak K, Kaminska D, Bartoszek D, Zabinska M, et al. The influence of non-HLA antibodies directed against angiotensin II type 1 receptor (AT1R) on early renal transplant outcomes. Transpl Int 2014;27:1029-38.

20. Lee J, Huh KH, Park Y, Park BG, Yang J, Jeong JC, et al. The clinicopathological relevance of pretransplant anti-angiotensin II type 1 receptor antibodies in renal transplantation. Nephrol Dial Transplant 2017;32: 1244-50.

21. Sanchez-Zapardiel E, Castro-Panete MJ, Castillo-Rama M, Morales P, Lora-Pablos D, Valero-Hervas D, et al. Harmful effect of preformed antiMICA antibodies on renal allograft evolution in early posttransplantation period. Transplantation 2013;96:70-8.

22. Caro-Oleas JL, Gonzalez-Escribano MF, Gonzalez-Roncero FM, Acevedo-Calado MJ, Cabello-Chaves V, Gentil-Govantes MA, et al. Clinical relevance of HLA donor-specific antibodies detected by single antigen assay in kidney transplantation. Nephrol Dial Transplant 2012;27:1231-8.

23. Lefaucheur C, Loupy A, Hill GS, Andrade J, Nochy D, Antoine C, et al. Preexisting donor-specific HLA antibodies predict outcome in kidney transplantation. J Am Soc Nephrol 2010;21:1398-406.

24. Harrison-Bernard LM, Navar LG, Ho MM, Vinson GP, el-Dahr SS. Immunohistochemical localization of ANG II AT1 receptor in adult rat kidney using a monoclonal antibody. Am J Physiol 1997;273:F170-7.

25. Kill A, Tabeling C, Undeutsch R, Kuhl AA, Gunther J, Radic M, et al. Autoantibodies to angiotensin and endothelin receptors in systemic sclerosis induce cellular and systemic events associated with disease pathogenesis. Arthritis Res Ther 2014;16:R29.

26. Win TS and Pettigrew GJ. Humoral autoimmunity and transplant vasculopathy: when allo is not enough. Transplantation 2010;90:113-20.

27. Stegall MD, Chedid MF, Cornell LD. The role of complement in antibodymediated rejection in kidney transplantation. Nat Rev Nephrol 2012;8: 670-8.

28. Cuevas E, Arreola-Guerra JM, Hernandez-Mendez EA, Salcedo I, Castelán N, Uribe-Uribe NO, et al. Pretransplant angiotensin II type 1-receptor antibodies are a risk factor for earlier detection of de novo HLA donor-specific antibodies. Nephrol Dial Transplant 2016;31:1738-45.

29. Chung BH, Joo YY, Lee J, Kim HD, Kim JI, Moon IS, et al. Impact of $\mathrm{ABO}$ incompatibility on the development of acute antibody-mediated rejection in kidney transplant recipients presensitized to HLA. PLoS One 2015; 10:e0123638.

30. Kim MG. Does the timing of acute rejection matter with the graft outcome in kidney transplantation? Kidney Res Clin Pract 2015;34:123-4.

31. Koo EH, Jang HR, Lee JE, Park JB, Kim SJ, Kim DJ, et al. The impact of early and late acute rejection on graft survival in renal transplantation. Kidney Res Clin Pract 2015;34:160-4.

32. Luo L, Li Z, Wu W, Luo G, Xu C, Sun Z, et al. Role of MICA antibodies in solid organ transplantation. Clin Transplant 2014;28:152-60.

33. European Renal Best Practice Transplantation Guideline Development G. ERBP guideline on the management and evaluation of the kidney donor and recipient. Nephrol Dial Transplant 2013;28(S2):ii1-71.

34. Lee H, Kim JI, Moon IS, Chung BH, Yang CW, Kim Y, et al. Investigation of serum angiotensin II type 1 receptor antibodies at the time of renal allograft rejection. Ann Lab Med 2015;35:314-20. 